\title{
Geschichte der Entstehung und erste Jahre der Arbeitsgemeinschaft für Osteosynthesefragen 1952-1963 (2)*
}

\author{
Urs F. A. Heim
}

\section{Strukturen, Arbeiten}

Maurice Müller hat immer gesagt, die AO beruhe auf 4 Säulen: Instrumentation, Forschung, Dokumentation und Unterricht (auf Englisch Instrumentation, Research, Documentation, Teaching). Die 4 Anfangsbuchstaben IRDT werden wir als Hinweis zu den Querverbindungen verwenden. Ich habe vor vielen Jahren von meinem Freund und Grafiker Klaus Oberli dazu ein kleines tempelartiges Gebilde zeichnen lassen (Abb. 21), das sich inzwischen einer gewissen Popularität erfreut (Ph.101, Erläuterung s.u.). Es ist eine Arbeitshilfe und erlaubt auch einer strengen Chronologie auszuweichen.

Der Blick wendet sich zunächst den getragenen Strukturen zu:

Zuoberst die Buchstaben A und O, welche ohne die verbindende Schleife auseinanderzufallen drohen. Die freundschaftliche, sehr weitgehende Zusammenarbeit innerhalb der Gruppe (Abb. 22) ist die Voraussetzung für den inneren Halt und dann den Erfolg. Die felsige Grundlage symbolisiert Strukturen und Regeln:

Das Recht erzwang eine Vereinsstruktur. Da alle gleichberechtigt sein mussten, wollte man keine Hierarchie (Abb. 23). Also keinen Präsidenten, sondern nur einen „Obmann“, primus inter pares. Dieser organisierte intern die Tagungen (23 pro Jahr) und war nach außen ein guter Repräsentant (Kontakte, Gäste, Einladungen etc.). Der Begriff Obmann ist in unserem Land uralt. Es war immer unbestritten, dass Robert Schneider diese Aufgabe übernehmen sollte.

* Teil 1 erschien im OP-JOURNAL 1/2012, Seite 98.

OP-JOURNAL 2012; 28: 280-288

(c) Georg Thieme Verlag KG Stuttgart · New York DOI http://dx.doi.org/10.1055/s-0032-1328341
Ein Vorstand war vorgeschrieben. Bis 1982 gab es jedoch nie Sitzungen. Alle Fragen und Probleme wurden mündlich im Rahmen der de facto existierenden „Kerngruppe“ (Abb. 24) vorbesprochen. Nach 1957 war auch Martin Allgöwer dabei. Kontroversen bei Abstimmungen und Wahlen waren deshalb extrem selten (ich mag mich nur an eine erinnern).

Sekretär (oder Aktuar) der Gruppe sollte der erfindungsreiche Maurice Müller sein, der immer mit neuen Ideen kam, Prototypen vorstellte (die noch lange nicht zu haben waren) und damit den Appetit der Mitglieder zur Unzeit anstachelte.

Martin Allgöwer sollte über die Finanzen als Kassier wachen, ein großes Problem für die Gruppe, die sich enorme Ziele gesetzt hatte und beim Start nur über persönliche Mittel verfügte. Anfänglich half der erfahrene Willenegger mit seinen Beziehungen, u. A. mit Betteln bei Versicherungen. Bald kam der befreundete Ökonom Dr. Peter von Rechenberg (19201992) (Abb. 25) in Chur dazu, welcher seither Fachmann in finanziellen Fragen wurde. Er erwies sich auch in Konfliktsituationen als wertvoller Vermittler.

Mit ihm hat die AO 1960 eine neue Struktur, die Synthes AG Chur, geschaffen. Diese sicherte das weitere stabile Wachstum und Einvernehmen mit den Produzenten. Darin waren die Ärzte Mehrheit, vom Gewinn jedoch aus ethischen Gründen ausgeschlossen. Es war dies eine neuartige Lösung, die vielfach bewundert wurde. Aus den Erträgen der Produktion sicherten Prozente Forschung, Unterricht und Dokumentation der AO.

Und nun zum Säulenquartett: alle 4 sind gleich in Form und Größe, es besteht kein Vorrang. Alle Beschriftungen sind austauschbar, sodass je nach Standort des Betrachters seine Lieblingssäule zuvorderst zu stehen scheint, es aber nicht

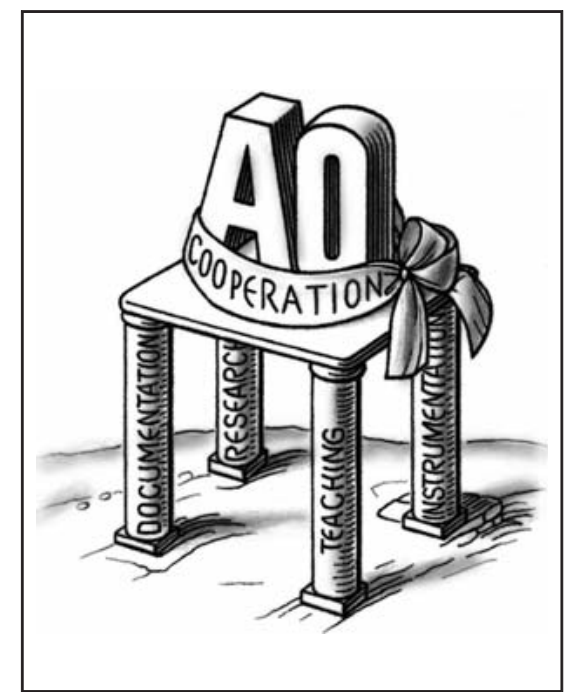

Abb. 21 Zeichnung K. Oberli/Heim Bern, ca. 1975. Symbol der Strukturen. Legende siehe Text.

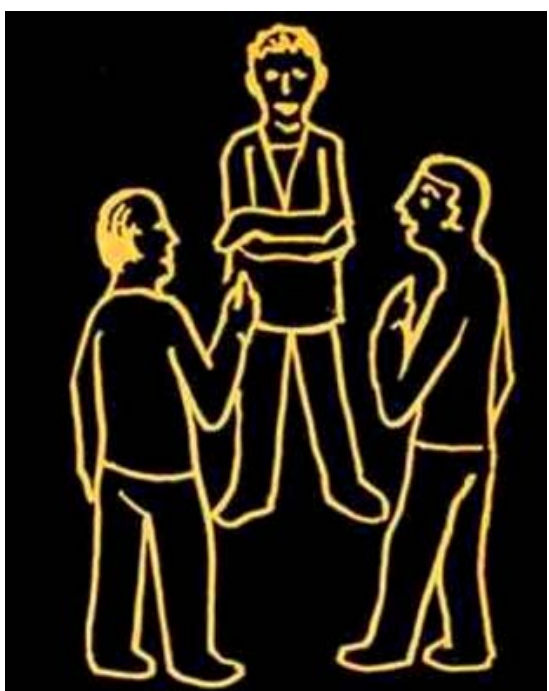

Abb. 22 Zeichnung K. Oberli/Heim. Schema der kommunikativ-kooperativen Struktur (AOTeamwork).

ist. Wird aber eine beschädigt oder gar entfernt, droht das Konstrukt zur Seite zu fallen und zu brechen. Die Reihenfolge, in welcher wir hier die einzelnen 4 ansehen, ist rein zufällig. Meine Auswahl 


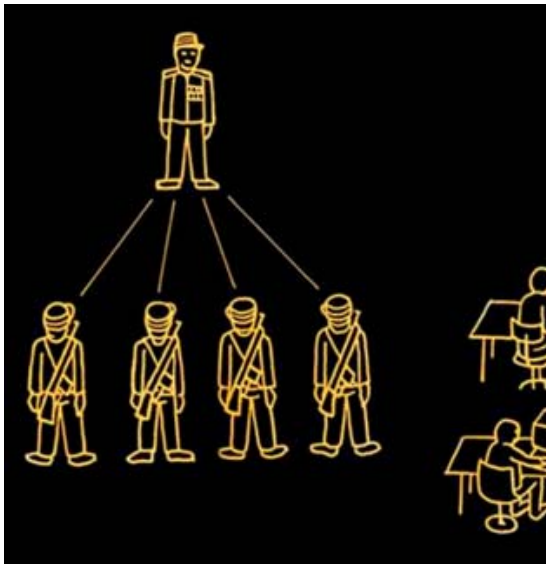

Abb. 23 Zeichnung K. Oberli/Heim. Die hierarchische Standardstruktur (Militär, Administration, Führung im großen Krankenhaus). Diese hätte den kleinen schweizerischen, auf Freundschaft aufgebauten Arbeitskreis verunmöglicht.

hat eventuell etwas mit der Chronologie und meinen ersten Kontakten mit der AO in Chur im Oktober 1958 zu tun:

\section{Die Säule Instrumentation (I)}

Maurice Müller hat mir einmal gesagt: „In der AO haben wir nichts Neues erfunden, sondern nur das Bestehende verbessert": ein Understatement.

Die zeitliche Folge der Entstehung der einzelnen Bestandteile der Instrumentation lässt sich kurz zusammenfassen:

Ende 1958 waren die neue AO-Kortikalis- und Spongiosaschraube mit Imbusinnensechskant und die dazugehörigen Instrumente bereits in den Spitälern. Prototypen der Platten wurden vermutlich bei der Gründung 1958 in Biel von Maurice Müller gezeigt, kamen im Laufe des Jahres 1959 in die Spitäler, zusammen mit den flexiblen Markraumbohrern. Der neue AO-Marknagel entstand in Zusammenarbeit mit Robert Schneider. Er sollte die Mängel des von Willenegger 1956 eingeführten Nagels nach Herzog beseitigen. Die T-Platte und die nachfolgenden sogenannten „Formplatten“" gehen auf Walter Bandi zurück. 1960 war das ganze Instrumentarium in den Mitgliederspitälern im Einsatz, aber noch nicht durchgehend dokumentiert (D).

Nur an den „Fixateur externe“, den Lambotte 1902 erdacht, benannt und 1913 perfektioniert hatte (Ph.152), glaubte Müller damals nicht. Seine Konstruktion erfolgte daher leider erst viel später.
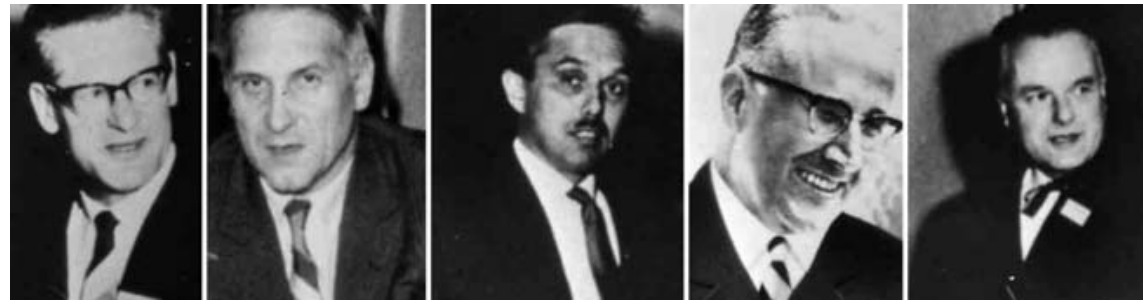

Abb. 24 Aus Schneider: 10 Jahre AO. op. cit. Die Kerngruppe der AO, von links Allgöwer, Willenegger, Müller, Bandi und Schneider (Die Aufnahmen stammen vom 1. Kurs in Davos Dezember 1960 [Namensschilder]. Bisher unveröffentlicht).

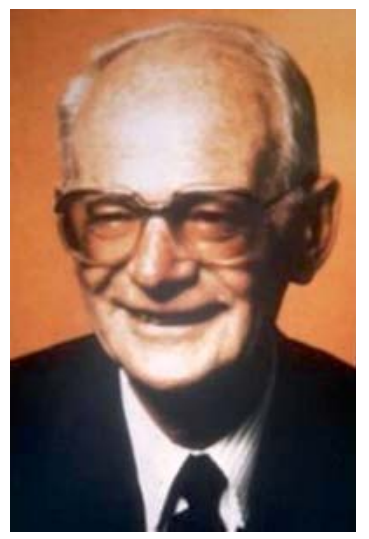

Abb. 25 Dr. Peter von Rechenberg. Wir danken der Familie für die Überlassung.

Dass ein neues, komplettes Instrumentarium in Konstruktion sei, wurde bald ruchbar. Für dessen Erwerb entstand aus einflussreichen Kreisen ein zunehmender Druck, obwohl die Erprobung noch nicht abgeschlossen war und die Produktion hinkend blieb.

Inzwischen waren bei den Implantaten vermehrt Korrosion und Plattenbrüche, dann auch Unregelmäßigkeiten aufgetaucht, also Probleme biomechanischer und metallurgischer Natur.

Deswegen kam es im Sommer 1960 zu Kontakten von Willenegger mit dem Physiker Prof. Dr. Ing. Reinhard Straumann (1892-1967) im Rotary-Klub Liestal. Dieser war Inhaber eines hoch qualifizierten, mit der Uhrenindustrie zusammenarbeitenden, metallurgisch-feinmechanischen Instituts in Waldenburg. Ihm gehörten auch weitere industrielle Betriebe. Es arbeitete bei ihm - auch in der Forschung - eine Gruppe von technischen Mitarbeitern mit Hochschulniveau: Séquin, Steinemann, Frau Pohler, der Sohn Fritz etc.

Straumann war zu einer Mitarbeit bereit, wollte aber auch bei der Produktion des neuen Instrumentariums beteiligt wer-

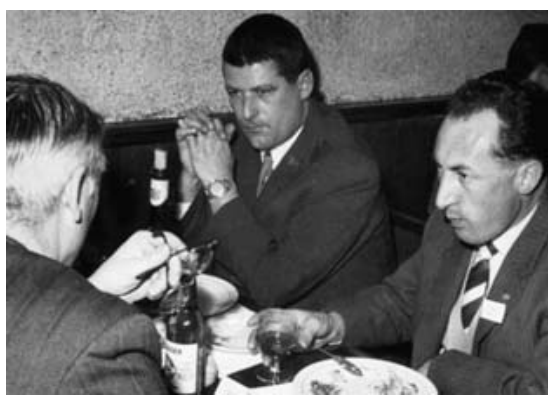

Abb. 26 Willenegger (von hinten) schlichtet Spannungen zwischen den Produzenten während des Essens an einem Kurs in Davos (1962?). Fritz Straumann in der Mitte. Ph. 93. Bisher unveröffentlicht.

den. Die mechanisch-metallurgische Entwicklung und Qualitätskontrolle konnte er optimal übernehmen.

Diese Entwicklung war für Mathys trotz der Überforderung, die auch ihm selbst bewusst war - schwer zu ertragen: sie stand im Widerspruch zur Vereinbarung von 1958. Es entwickelte sich deshalb ein hartnäckiges Misstrauen zwischen den Firmen und gegenüber den Ärzten (Abb. 26).

Nach längerem Hin und Her kam es 1963 zu einem Vertrag zwischen den beiden Produzenten, wobei die weltweiten Absatzgebiete zu bestimmen waren. Die zum Teil unbegreiflichen Aufteilungen derselben beruhen auf dem damals gewählten Losentscheid.

1961 war von Müller die technische Kommission gegründet worden. Sie hatte alle neuen Ideen und Prototypen vor einer Serienfabrikation zu prüfen. Im Rahmen einer ärztlichen Mehrheit mussten die technischen und wirtschaftlichen Möglichkeiten und Bedürfnisse der Produzenten berücksichtigt werden.

Im Frühjahr 1962 (schneller als vorgesehen) wurde das Instrumentarium in den 

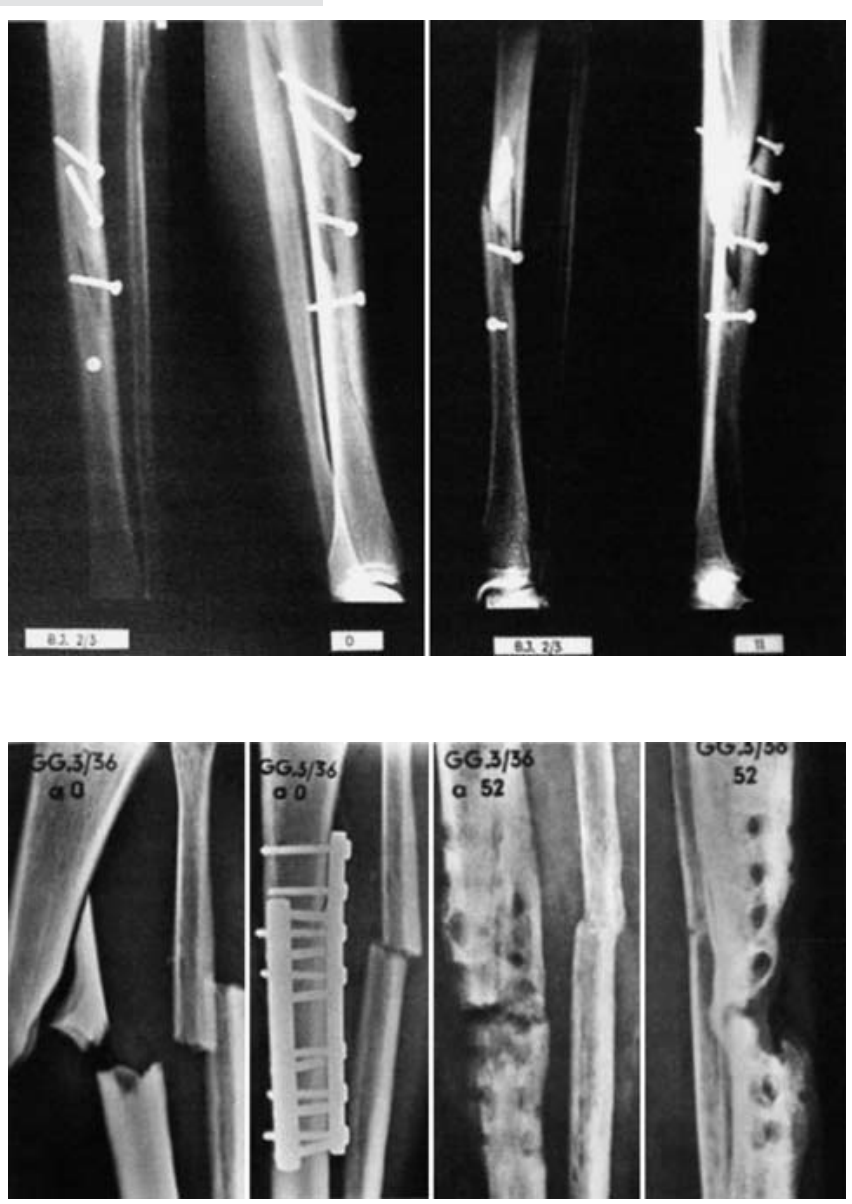

Abb. 28 Dokumentation eines Misserfolgs 1962: Nach Doppelplatte Infektion und Nekrosen infolge Devitalisation. Diese Technik wurde aufgrund solcher Resultate wieder ganz verlassen. Ph. 165. freien Verkauf gegeben. Man versuchte, das Risiko von Fehlleistungen durch verschiedene Formen der Ausbildung (T) möglichst tief zu halten.

\section{Die Säule Dokumentation (D)}

Für die $\mathrm{AO}$ und ihre begreiflicherweise stark angefochtenen Theorien - besonders die von Danis übernommene Wünschbarkeit einer kallusfreien, sogenannt „primären“ (später in „direkte“ umbenannte) Frakturheilung - fehlten anfänglich wissenschaftliche tierexperimentelle Unterlagen praktisch vollständig. Zum Nachweis dieses unglaubwürdigen Vorgangs war man ausschließlich auf den Verlauf im Röntgenbild angewiesen.

Jedes Mitglied hatte sich daher verpflichtet, bei allen operierten Patienten den Verlauf zu asservieren und zentral auswerten zu lassen. Einzusenden waren Röntgenbilder von Unfall, Osteosynthese, nach 4 und 12 Monaten. Die entsprechenden klinischen Befunde wurden auf standardisierten sogenannten „CodeBlättern“ (anfänglich von Hand, später mit EDV) notiert und bearbeitet.
Dieser Teil der Dokumentation war nie fehlerfrei. Er gab häufig zu Diskussionen und Ärger bei den Tagungen Anlass.

Störungen der Heilung waren im Röntgenbild nach 4 Monaten erkennbar und dann noch aufzufangen. Nach 1 Jahr sollte die Konsolidation abgeschlossen und die volle Funktion wiederhergestellt sein.

Wichtig waren vor allem die Verläufe bei Prototypen und bei Komplikationen (Motto: „Aus den Fehlern lernen“) (Abb. 27, 28). Diese wurden intern ausgiebig diskutiert und die Ergebnisse in den - laufend überarbeiteten - sogenannten „Merkblättern“ (T) an die Mitglieder verteilt.

Technisch wurde nach dem von Maurice Müller bereits 1950-1952 in Fribourg ausgedachten System vorgegangen: die Röntgenbilder wurden mit einer LeicaKamera aufgenommen. Vom Negativ wurden immer 2 Kleinbildkopien (also Format $1: 1$ ) angefertigt und auf feste Karten aufgeklebt. An diesen wurden nach den Angaben der Code-Blätter Randlochungen vorgenommen für die spätere Auswertung. Je eine Karte ging

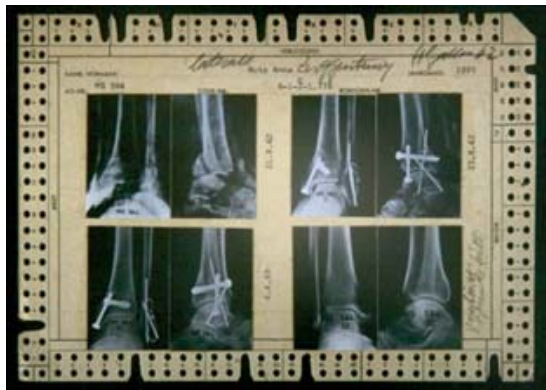

Abb. 29 Beispiel einer Dokumentationskarte mit aufgeklebten Röntgenbildkopien und Randlochung. AO-Dokumentation Davos 1960, unveröffentlicht.

mit den Röntgenbildern an den Operateur zurück (Abb. 29). Das gab diesem einen ständigen Überblick über das eigene Krankengut. Die 2. Karte blieb zentral archiviert und stand jedem Mitglied zur Einsicht und Bearbeitung bereit. Die Negative sind bis heute erhalten, ebenso Tausende von früheren Karten.

Die Bedeutung der Dokumentation für die Glaubwürdigkeit ihrer Technik war allen bewusst, alle machten mit (mit Ausnahme der Senioren). Sie war sehr arbeitsintensiv und teuer: die Mitglieder mussten anfänglich selbst dafür bezahlen (Fr. 18,00 pro Fall). Später ging die Finanzierung über Synthes.

Im 1. Jahr (Sommer 1959-Sommer 1960) waren an die 1000 (noch nicht abgeschlossene) Osteosynthesen erfasst, bis Ende 1963 waren es ca. 6000 (Ph. 157203). Lückenlos war das System sicher nie. Die Code-Blätter sind verschollen. Aus unvollständigen Röntgenbildserien können aber Prototypen erkannt werden, ebenso der ungefähre Zeitpunkt der Serienfabrikation neuer Implantate. In den größeren Spitälern lassen sich „Lieblingsverfahren“ erkennen (Indikation und Technik waren ja den Mitgliedern freigestellt). Eindeutig sind Trends zum Marktnagel bei Willenegger und Schneider, für die Platte bei Müller und die Kombination Platte/Schrauben für Allgöwer und Bandi. In mehreren Spitälern wurde aber immer das ganze Krankengut systematisch dokumentiert, was die Publikation mehrerer geschlossener Serien möglich machte (Ph.112$115,129)$.

Enge Beziehungen bestehen zwischen Dokumentation und Klassifikation. Die Dokumentation ist auch unverzichtbar bei der Einführung neuer Operationsverfahren oder Implantaten, auch im Tierversuch (R). 


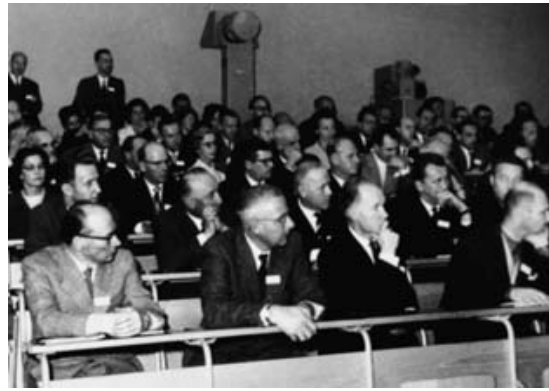

Abb. 30 Hörsaal des Instituts für experimentelle Chirurgie anlässlich des 1. AO-Kurses November 1960 in Davos (AO-Diasammlung).

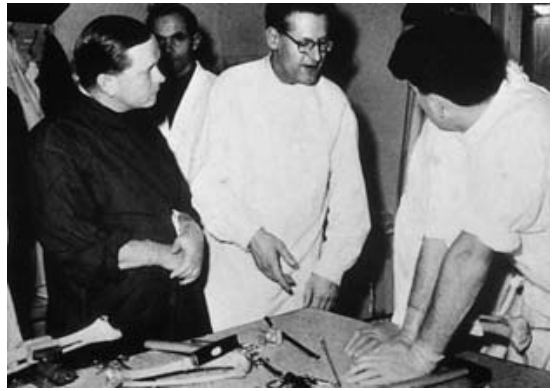

Abb.31 Bei den praktischen Übungen der Ehrengast von 1961 John Charnley mit Martin Allgöwer an den Arbeitsplätzen (Ph. 88).

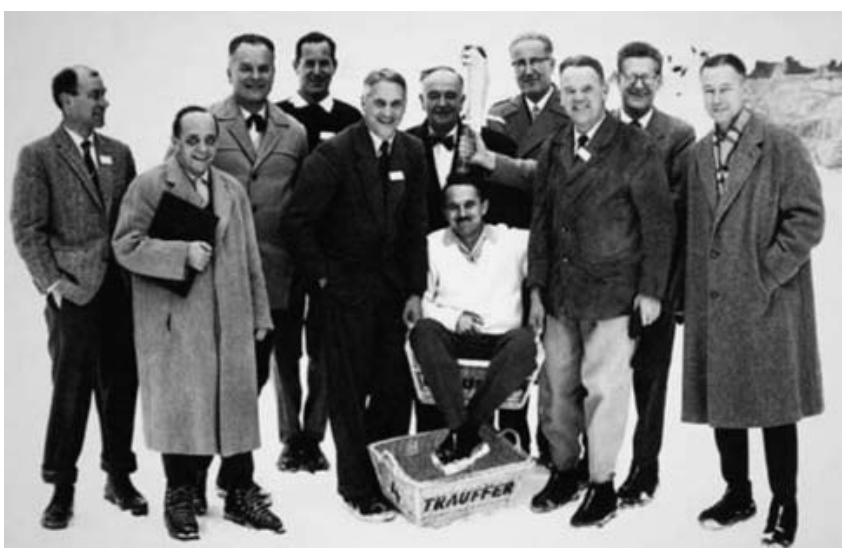

Abb. 32 Freudiges Zusammensein der Referenten und Instruktoren am Ende des 1. AO-Kurses. Im Bild neue Mitwirkende und Mitglieder: Bloch rechts (Mitglied 1959), Fischer und Keller links (Mitglieder 1961). In der Mitte Müller sitzend. Wird fälschlicherweise oft als Gründungsbild der Mitglieder von 1958 bezeichnet und gezeigt (AO-Diasammlung).

\section{Die Säule Unterricht (Teaching) ( $T$ )}

Dem Teaching gegenüber befindet sich immer das Learning. Das Neue wird gelernt und dann Bewährtes weitergegeben.

- Intern geschah dies in der AO mit Diskussion und Redaktion der sog. „Merkblätter“. Diese wurden nach den Tagungen entsprechend optimiert. Sie bildeten - z.T. wörtlich - die Unterlagen für das 1. AO-Buch 1963 (Abb. 35)

- Externer Unterricht war gefordert, sobald die Instrumentation (I) über den Kreis der Mitglieder ausgegeben wurde.

- Dass ein Buch allein als Unterrichtsmittel nicht taugt, hatte man bei Lambotte, Danis und selbst bei Böhler gesehen.

- Von 1960 an wurden - zuerst in Davos - 4- bis 5-tägige Kurse durchgeführt. Muster dafür waren die von vielen Schweizer Chirurgen besuchten handchirurgischen Kurse von Marc Iselin in Paris-Nanterre. In diesen wurden in praktischen Übungen alle Standardoperationen von den Teilnehmern an Leichenhänden ausgeführt und LiveOperationen an Patienten über Fern- sehen (damals nur schwarz-weiß, 4× vergrößert) in ein Auditorium übertragen. Im Juni 1960 traf ich dort Maurice Müller und auch spätere AO-Mitglieder.

- In den AO-Kursen alternierten Vorträge und Diskussionen über Prinzipien und Techniken (Abb. 30) (später auch über wissenschaftliche Themen) mit praktischen Übungen (Abb. 31) an Leichenknochen. Diese Übungen wurden zentral durch Fernsehen geleitet und in die Übungsräume projiziert. Trotz gewisser Mängel waren die Kurse ein großer Erfolg und die Freude des Teams über das Gelingen des Ersten besonders groß (Abb. 32).

Es hatte sich gezeigt, dass in den Unterricht auch das Operationspersonal einbezogen werden musste. Diese Mitarbeiterinnen waren bisher nur für relativ wenig Material verantwortlich. Sie bekamen nun so viel Neues an Implantaten und Instrumenten zu kennen, zu gebrauchen, zu reinigen und nachzubestellen, dass sie Managerinnen eines „Eisenwarenladens" wurden. Ein spezieller Kurs für Operationsschwestern wurde 1961 nach dem Chirurgenkurs durchgeführt und seither jährlich wiederholt. Martin

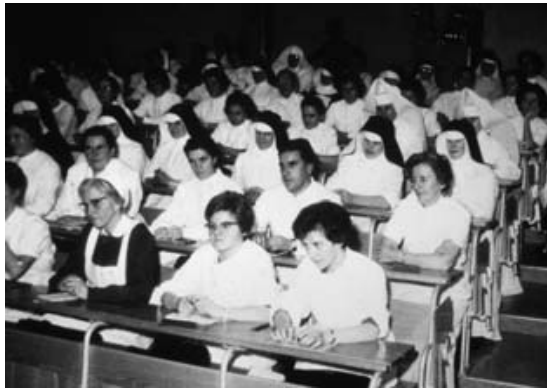

Abb.33 Damalige Operationsschwestern am Kurs 1961 im Hörsaal...

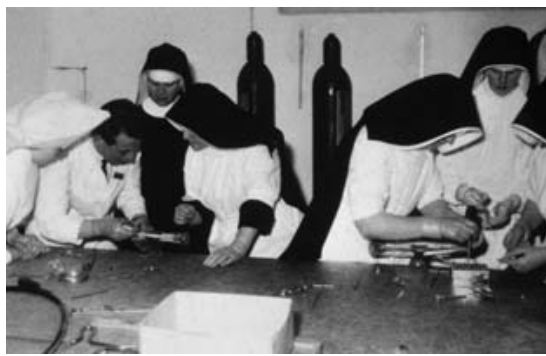

Abb. 34 .... und bei den praktischen Übungen (AO-Diasammlung).

\section{TECHNIK DER OPERATIVEN FRAKTURENBEHANDLUNG}

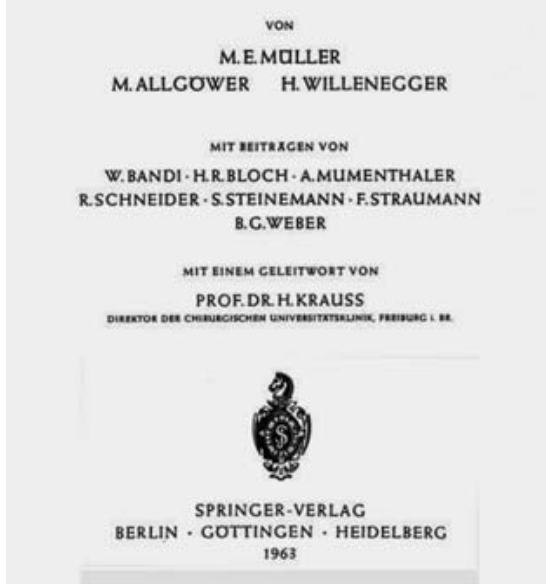

Abb. 35 Titelblatt des 1. AO-Buches 1963, (Ph. 96), Library of Congress Catalog Number 63-17394.

Allgöwer und Robert Schneider organisierten und leiteten diese persönlich (Abb. 33, 34).

- Wirksamer für das Learning war die länger dauernde Mitarbeit ausländischer Gastärzte in AO-Spitälern. Das System wurde später von Willenegger offiziell als „AO-Stipendium“ von 2 bis 3 Monaten Dauer, vor allem für Kolle- 


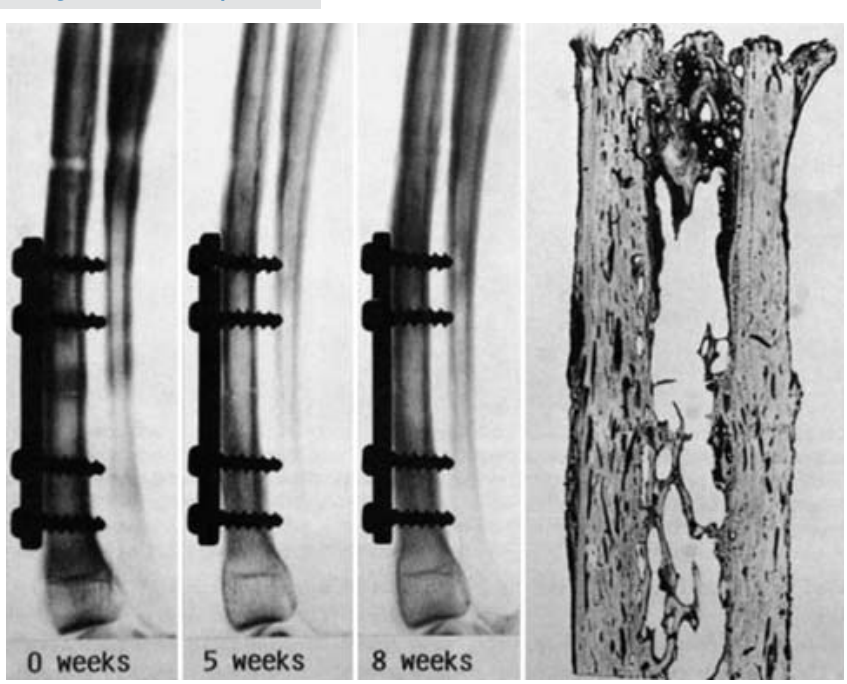

gen aus Entwicklungsländern, standardisiert. Diese konnten mit einem Touristenvisum einreisen. Viele dieser Stipendiaten erhielten später in ihren Ländern leitende Stellungen und trugen viel zur Verbreitung der Ideen der AO bei.

- Das 1. Buch der AO „Die Technik der operativen Frakturenbehandlung“ (Abb. 35) [10] erschien 1963 bei Springer in Heidelberg. Daran hatte man schon früh gearbeitet, nebst der Kerngruppe weitere Mitglieder und jüngere Mitarbeiter. Es enthält theoretische Einführungen, technische Angaben und dient auch nach Kursbesuch zum Nachschlagen. Darin sind auch die ersten Ergebnisse der experimentellen Arbeiten zu finden (R). Abschließende Kapitel über offene Frakturen, Infekt und Schock weisen auf Allgöwer, sein Team und Willenegger hin (Ph. 96, 150 ff.). Die darin angegebenen histologischen Untersuchungen von Wagner und Schenk/Willenegger wurden 1963 und 1964 publiziert und sind in Ph. 129 im Detail angegeben.

Das Buch konnte allerdings nur deutschsprachigen Lesern dienen. Die 1965 erschienene gekürzte englische Ausgabe ist praktisch vergessen.

- Erst das „AO-Manual“ [11], erschienen 1969, bekam mit Übersetzungen und weiteren Auflagen internationale Verbreitung.

\section{Die Säule Forschung (Research) (R)}

Am 1. Juni 1959 konnte das „Laboratorium für experimentelle Chirurgie“ in Davos den Betrieb aufnehmen. Es wurde schon früh von Gästen aus nah und fern besucht.
Abb. 36 Experiment (Aus: Schenk R./Willenegger H.: Zur Histologie der primären Knochenheilung. Langenbecks Arch. 308, 4401964 [Ph. 121]).

Anfänglich war der personelle Aufwand für die zentrale Dokumentation (D) groß, aber als Voraussetzung für öffentliche Auftritte und Kurse unerlässlich.

Experimentell wurden im Team Verbrennung, hämorrhagischer Schock, Blutvolumen, zentraler Venendruck etc. bearbeitet. Das Churer Team war dazu im Turnus in Davos (Burri, Gruber, Frau Siegrist, Segmüller, Matter).

Biomechanische, histologisch-experimentelle Untersuchungen begannen zuerst an der Orthopädie Münster und in Basel:

Heinz Wagner (1929-2001) zeigte 1961, dass korrekt im Knochen eingeführte AO-Schrauben unter Druck stabil bleiben und osteogenetisch wirken (Ph.120). Langenbecks Arch. 305, 28-40, 1963.

Dem Team Robert Schenk (1923, Anatom) und $\mathbf{H}$. Willenegger gelang wenig später der Nachweis des direkten kortikalen Durchbaus des Havers'schen Gefäßsystems mit einem Osteotomiemodell unter Plattenkompression (Abb. 36).

Die dazu notwendige extrem dünne Säge (0,2 mm) wurde von Fritz Straumann (1921-1988) (Abb.26), dem Sohn von Prof. R. Straumann, in der firmeneigenen Staubsaugerfabrik angefertigt. Das war der Beginn seines lebenslänglichen wissenschaftlichen Engagements bei der AO.

1961 wurde in Davos von den gleichen 3 Autoren das Schaf als bevorzugtes Versuchstier eingeführt.

1962 begannen die biomechanischen Untersuchungen von Perren (mit dem
Orthopäden Huggler). Er verbrachte jeweils von Chur aus 2 Wochentage in Davos und verwendete zum Nachweis des Verlaufs der Druckwerte nach der Osteotomie Dehnungsmessstreifen (strain gauges). Er beschrieb dabei eine neue Morphologie an der der Platte gegenüberliegenden Kortikalis - die „Spaltheilung“, wo im Gegensatz zur „Kontaktheilung“ zuerst ein Saum lamellären Knochens entsteht. Dieser wird dann nachträglich vom Havers'schen System umgebaut (in Ph. 122 f abgebildet).

In der Folge zeigte sich, dass in der Klinik die direkte Frakturheilung seltener und weniger resistent ist als die indirekte.

Andere Forschungsprogramme betrafen metallurgisch-physikalische Probleme (z.T. in Waldenburg ausgeführt) vom dortigen akademischen Team (Steinemann, Séquin, Frau Pohler): Über Korrosion, Ermüdungsbrüche von Implantaten, Materialfehler, Implantatoberfläche und dann die Suche nach neuen Legierungen für ihre physikalischen Eigenschaften und die Biokompatibiliät (I).

\section{Etappen zur Anerkennung (1960-1963)}

Öffentliche Auftritte waren nun dank Fertigstellung des Instrumentariums (I) und genügenden Langzeitresultaten der Dokumentation (D) im Jahr 1960 möglich geworden.

- Zuerst ging es nach Deutschland: in Freiburg i. Br. fand am 4.-5. März unter Leitung von Prof. Hermann Krauss, Ordinarius für Chirurgie, eine Unfalltagung (der Deutschen Berufsgenossenschaften?) statt.

- Wegen der Zusammenarbeit im Bluttransfusionswesen war Krauss mit Prof. L. Heilmeyer (1899-1969), Ordinarius für Innere Medizin und Hämatologe, in ständiger Zusammenarbeit, dieser wiederum mit Willenegger, der ihn über die AO-Aktivitäten informierte. So kam es, dass Krauss Müller und Willenegger zu Vorträgen zu dieser Tagung einlud. Willenegger brachte gleich noch 7 Patienten aus Liestal mit. Krauss war ernsthaft interessiert. Er selbst oder Mitarbeiter nahmen fortan an den AO-Veranstaltungen teil: zuerst Koslowski, dann Siegfried Weller, welchem die dornenvolle Aufgabe zufiel, Instrumentarium und Ersatzteile nach Deutschland einzuschmuggeln und die DokumentationsRöntgenbilder hin und herzuschieben. 
- Auf der gleichen Tagung demonstrierte Müller in einem Nebenraum das Instrumentarium: Jörg Böhler.

Der Sohn von Lorenz und damals Primarius im neuen Unfallkrankenhaus Linz ließ sich alles genau erklären und meldete sich gleich für die bevorstehende (18.19. März) AO-Tagung in Interlaken an, wo die ersten Langzeitergebnisse der Dokumentation vorgestellt und die Vorträge für den Chirurgenkongress vorbesprochen wurden. Mit Jörg Böhler war eine 1. solide Brücke ins östliche Nachbarland entstanden.

- Am jährlichen Kongress der Schweizerischen Gesellschaft für Chirurgie (16.-17. Mai in Genf) gelang es dem abtretenden Präsidenten der AO, Patry, ganz am Ende 4 Kurzvorträge ohne Diskussionszeit zuzuteilen. Das Interesse war aber so groß, dass für das Thema eine

- Sondersitzung beschlossen wurde. Diese fand am 24. November 1960 in Bern statt. Vonseiten der AO hielten Vorträge: Müller, Willenegger, Schneider und Bandi (der den erkrankten Allgöwer auch in der Diskussion ersetzte). Es ging schließlich um die grundsätzliche Frage konservative oder operative Frakturbehandlung. Die mit der ausführlichen Dokumentation von der AO postulierte „kallusfreie“"Konsolidation war begreiflicherweise unglaubwürdig. Experimentell war dazu erst eine komplizierte Versuchsanordnung von Bassett aus New York (R) vorhanden, während aus der Berner Klinik Versuchsmodelle vorgelegt wurden, welche früh reichlich und ausschließlich periostalen und endostalen Kallus zeigten. Die Tagung wurde immer unsachlicher und endete im Durcheinander (Ph. 71).

Ein letzter Vortrag von Dr. E. Bauer von der Schweizerischen Unfallversicherungsanstalt (SUVA), der bereits aufgrund der Zahlen die Vorteile der AOTechnik für die Versicherung (Arbeitsausfall, Invalidität) nachwies, blieb daher unbeachtet.

\section{Die A0-Kurse in Davos}

Der erste Kurs fand vom 11.-15. November 1960 im Institut für experimentelle Chirurgie statt. Über dessen Struktur etc. wurde bereits berichtet (T) (S. 283). Das Interesse dazu war von allen Seiten sehr groß. Statt der eingeplanten 25 Teilnehmer waren schließlich 102 Personen anwesend, 46 aktive Schweizer Chirurgen und 13 ausländische Kollegen, darunter 4 Deutsche und 1 Wiener Ordinarius. Es entstanden auch sehr fruchtbare Beziehungen zu Dr. H. Rosen (1930-2000), Orthopäde in New York, ferner zu den Orthopäden in Nizza.

Unglücklicherweise hatte man im Voraus versprochen, dass die Teilnehmer nach dem Kurs das heiß ersehnte Instrumentarium erwerben könnten. $\mathrm{Zu}$ diesem Zeitpunkt waren dann aber erst 16 neue Sets vorhanden. Es gab auch noch andere personelle, logistische und organisatorische Probleme.

Man hatte zu diesem Ereignis einen Ehrengast in der Person von Prof. Hermann Krauss (Abb. 37) aus Freiburg eingeladen. Er war der einzige Direktor einer Universitätsklinik, der zur AO positiv eingestellt war und sein ganzes Team engagierte.

Ein Ehrengast wurde in der Folge zu jeden Kurs eingeladen. Es sollte sich um eine auswärtige Persönlichkeit von internationalem Ansehen handeln, die der AO gegenüber durchaus kritisch gegenüberstehen konnte. Man erwartete vom Gast einen Vortrag zu einem Thema eigener Wahl.

Am 2. Kurs, der Anfang Dezember 1961 stattfand, wurden 136 Teilnehmer registriert. Müller hatte 2 Ehrengäste eingeladen: seinen Mentor und Freund John Charnley (Abb. 31) aus Wrightington (GB), der die Kompressionsarthrodese eingeführt hatte und als erster eine Totalprothese des Hüftgelenks erdachte, und Prof. Michel Postel, Schüler und Nachfolger des berühmten Orthopäden Prof. Merle d'Aubigné aus Paris. Leider stellten sich mit dem Letzteren dann keine Beziehungen von Dauer ein.

1962 waren alle Mitglieder mit den Vorarbeiten des AO-Buches so intensiv beschäftigt, dass die Energien nicht für einen Kurs reichten. Dafür waren sie mit vielen Vorträgen am Kongress der Mittelrheinischen Gesellschaft für Chirurgie präsent und Robert Schneider war mit Vorzeigeoperationen und Vorträgen in Deutschland gebunden.

1963 kam auch die Einladung zum Deutschen Chirurgenkongress in München.

Der 3. Chirurgenkurs fand erst wieder 1963 auf speziellen Wunsch aus Deutschland statt (24.-27. März), diesmal mit 113 Teilnehmern. Ehrengast war Prof. H. Bürkle de la Camp, emeritierter Direktor des „Bergmannsheil“, der ältesten und ehrwürdigsten deutschen Unfallklinik. Sein Mitmachen am Kurs zeigte die gewonnene positive Einstellung der Berufsgenossenschaften an - für die Ideen der AO ein großer weiterer Gewinn in Deutschland.

Ein 4. Kurs (der 2. des Jahres) wurde Ende 1963 in den Fremdsprachen Englisch und Französisch abgehalten.

\section{Neue Mitglieder}

Zwischen November 1958 und Ende 1962 wurden 10 neue Mitglieder aufgenommen:

- Hans Rudolph Bloch (1913-2003), Chefarzt in Glarus bis 1973 (Abb. 32, außen rechts neben Allgöwer).

- Ernst Kaiser (1903-1967), Chefarzt des Stadtspitals Waid in Zürich, viszeralchirurgisch tätig, überließ die Traumatologie ganz seinem Mitarbeiter

- Max Landolt (geb. 1925). Dieser war dann bei allen Tagungen und Kursen dabei. Er war ein fundierter Kenner der Böhler-Schule, wo er 8 Monate gearbeitet hatte.

- Clemente Molo (1909-1998), Chefarzt in Bellinzona.

- Irwin S. Leimbach (1890-1985). Orthopaedic Consultant beim US-Expeditionscorps. Mehrmals bei Müller in St. Gallen.

- Reinhard Fischer (geb. 1920) (Abb. 32 , links ganz außen), Chefarzt in Wattwil. War schon beim 1. Kurs 1960 und anschließend regelmäßig Referent und Instruktor.

- Otto Keller (1911-71) (Abb. 32 neben Fischer, mit Mappe), Chefarzt in Walenstadt. War ein Freund von Fischer.

- Willy Stähli (1915-1995), Chefarzt in Thun (1949-1980).

- Hermann Krauss (1897-1971), Mitarbeiter von Sauerbruch vor dem Krieg und wie dieser vor allem Viszeralchirurg. 1952 Ordinarius in Freiburg i. Br. Lernte die AO 1960 kennen (S. 284). Sein ganzes Team wirkte in der AO mit und bildete die Keimzelle für die in Deutschland allmählich entstehenden Unfallabteilungen in den größeren Krankenhäusern. Dazu gehörten damals:

- Fritz Kümmerle (geb. 1917). Wurde Ordinarius für Chirurgie in Mainz. Als Traumatologe nahm er dorthin

- Karl Heinz Schweikert (1929-1979) mit. Dieser begründete die Mainzer Unfallchirurgischen Symposien. 


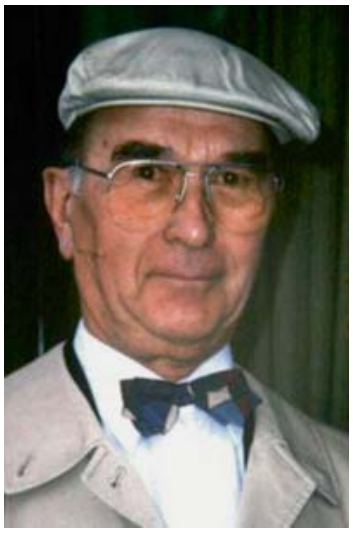

Abb. 37 Emmanuel Trojan (Foto U. Heim, ca. 1998).

- Jörg Rehn (1918-2008), später Chefarzt im „Bergmannsheil“ in Bochum, der ältesten Unfallklinik Deutschlands.

- Leo Koslowski (1921-2008), wurde Ordinarius für Chirurgie in Tübingen.

- Siegfried Weller (geb. 1928), bereits erwähnt (S.285), später Direktor der BG Unfallklinik Tübingen und langjähriger Obmann der Deutschen AO.

- Bernhard Barraud (1916-1998), Chefarzt in Aarberg.

- Urs Heim (geb. 1924), kam Oktober 1958 in das Kader von Martin Allgöwer, den er von Basel her kannte. Chefarzt am Kreuzspital Chur 19611981, Habilitation 1974. 1988-1993 Präsident der AO-International.

Er war 1962 das 1. Mitglied aus der „2. Generation“, d. h. selbstständig gewordener Schüler eines Gründers. Nach ihm wurde eine Pause in den Aufnahmen beschlossen (bis 1965).

Hier endet die Chronik. Die Gründer selbst haben das Jahr 1963 als Abschluss einer 1. Periode empfunden (Ph. 98): Die Strukturen hatten sich bewährt. Die ernstesten Widerstände waren vorüber. Sie hatten den inneren Zusammenhang gefestigt. Bei Robert Schneiders Vorträgen blieben nun Pfeifkonzerte bei Metall in Röntgenbildern aus. Maurice Müller war nach Bern berufen worden. Martin Allgöwer wurde ao. Professor. Das legitimierende Buch war erschienen.

Es folgte die Expansion, die nicht mehr Gegenstand dieser Studie ist.

\section{Rückblick und Ausblick}

Endständig möchte ich noch einige persönliche Überlegungen beifügen:

\section{Verdanken und Gedenken}

Der sogenannte „AO-Geist“ hat über die Jahrzehnte eine ganze Fülle spontaner bedeutender Aktionen - vor allem für den Unterricht - hervorgebracht. Es mögen hier einige wenige Beispiele genügen:

- Hans Willenegger zeigt im „missionarischen“ Einsatz ein unnachahmlich selbstsicheres Verhalten auf den Bildern, wo er mit Würdenträgern oder Staatsoberhäuptern zweifelhafter Dignität Hände schüttelt. Für den Unterricht der AO sollten keine politischen Barrieren gelten.

Während Reisen für Kurse in ferne Kontinente ließ er jeweils die Mitreferenten im Flug zu sich kommen, korrigierte ihre Vorträge und die Anordnung ihrer Diapositive.

In den Entwicklungsländern sprach er vordergründig über Indikation und Technik der Synthesen von Pseudarthrosen. Wegen der Risiken bei mangelhafter Technik oder Asepsis hütete er sich, die lokale, oft zweckmäßige konservative Frakturenbehandlung zu kritisieren.

- Prof. Emmanuel Trojan (geb. 1917) (Abb. 37) übernahm nach seiner Emeritierung in Wien als Vizepräsident der AO-International die Betreuung aller Länder jenseits des Eisernen Vorhangs. Noch in hohem Alter reiste er unter beschwerlichen Bedingungen mitten im Winter nach Sibirien, um dort AO-Kurse zu leiten.

- Rigmor Texhammar (Abb. 38) hat jahrelang die Kurse für Operationsschwestern organisiert, optimiert und weltweit selber geleitet. Bei diesen Arbeiten musste sie sich als Frau in Benehmen und Kleidung den Gebräuchen der besuchten Länder anpassen, was vor allem im islamischen Kulturbereich sehr beschwerlich war. Ihre erschöpfende Arbeit mit unendlich vielem Hin-und-her-Reisen über die Kontinente führte sie unter bescheidenen Anstellungsbedingungen aus. Als Erstautorin verfasste sie auch die 2. Auflage von „Das AO-Instrumentarium“ [12]. Endlich konnte sie - ihrem Wunsch entsprechend - wieder in ihr heimatliches Schweden zurückkehren. Ihr ist zu danken.

- Ann Murphy (Abb. 38), ihre sehr beliebte Nachfolgerin, welche Urs Heim in Salzburg als Stipendiatin gefunden und gemeldet hatte. Rigmor hat sie dann definitiv erwählt und eingeführt. Sie trug mit dem gleichen Einsatz diese große Last weiter, wurde uns aber leider nach wenigen Jahren durch eine

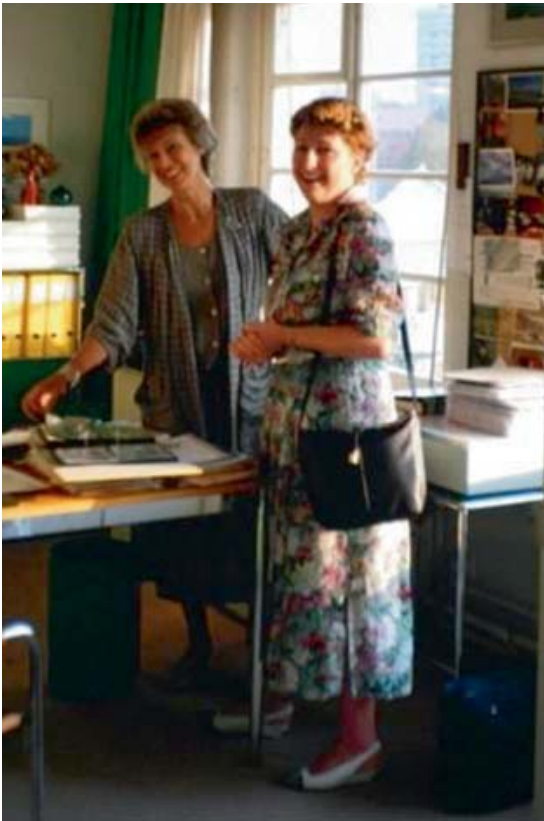

Abb.38 Amtsübergabe von Rigmor Texhammar an Ann Murphy 1999 (Foto U. Heim).

heimtückische Krankheit entrissen. Ihrer sollte man gedenken.

\section{Wie wurde die AO von den jungen Ärzten damals erlebt?}

Es war eine Art Erlösung aus der standardisierten schwerfälligen Routine hinein in echte aktive Notfallchirurgie. Die ständig sich vermehrenden technischen Möglichkeiten lösten Begeisterung aus, manchmal auch Übermut.

Es entstand eine Zäsur im ärztlichen Verhalten: der Patient wurde vom Operateur persönlich untersucht und informiert, dann seine Fraktur in einem einzigen Arbeitsgang definitiv versorgt. Die im konservativen System oft nötigen Korrekturen und Umdispositionen fielen weg. Die Aufmerksamkeit galt der Reposition und der Stabilität.

Für den Patienten war der Unfall eine Katastrophe, die seine unmittelbare Lebensweise traf und mittelfristige Pläne vernichtete. Er wurde „gefangen“ und kam in die Hände Fremder. Das entstandene moralische Tief dauerte früher Monate (bis zum sichtbaren tragfähigen Kallus). Die Notfalloperation markierte deren Ende. Von da an sollte es ja wieder aufwärts gehen.

Mitzusehen, wie er am nächsten Tag durch selbstgesteuerte Bewegung seine „Freiheit“" zurückbekam, war für uns Ärzte ein unerhörtes Erlebnis, für den Patien- 
ten nur eine freudige Überraschung. Nur wer früher einmal die lang dauernde Immobilisation in Gips oder Extension selbst erlebt hatte, konnte vergleichen. Pläne machen wurde jetzt wieder realistisch. Dass es moralisch wieder aufwärts ging, musste sich für Trophik und Zirkulation positiv auswirken und indirekt auch die Konsolidation anregen - so dachten wir.

Dieser krasse Unterschied zwischen den beiden Systemen ist nur noch den wenigen alten Ärzten in Erinnerung, welche vor den 60er-Jahren des letzten Jahrhunderts diplomiert wurden. Schon die jetzt Emeritierten haben diese Phase nicht mehr erlebt, höchstens in humanitären Einsätzen in Drittweltländern.

\section{Wie reagierten die Patienten?}

In den ersten Jahren - bis ca. 1961 - war eine gewisse Unsicherheit gegenüber der Operation bei den Notfallaufnahmen spürbar. Dass für Frakturrepositionen eine Narkose unerlässlich war, wusste jedermann. Alle rechneten auch entweder mit Gips oder „Streckbett“ und der entsprechenden Heilungsdauer.

Der Vorschlag einer Operation war hingegen neu, wurde aber meist durch den vertrauensbildenden unmittelbaren Kontakt mit dem Operateur akzeptiert. Bei ausländischen, fremdsprachigen Verunfallten war es schwieriger, besonders wenn sie aus Ländern kamen, in denen man ganz anderes gewohnt war.

So musste ich mich im Winter 1959/60 als Oberarzt während mehreren Stunden mit einer zutiefst erschrockenen Londoner Kollegin aus höheren gesellschaftlichen Kreisen befassen, die Martin Allgöwer - unter Druck weiterer Notfälle etwas kurz abgetan hatte. Sie willigte schließlich ein, obwohl ihr telefonisch kontaktierter „Orthopaedic Surgeon of the Queen" eindringlich abgeraten hatte. Wir wurden dann lebenslängliche Freunde.

Schon 1 Jahr später war das Eis gebrochen, „man“ war informiert. Nachdem eine junge Patientin einer befreundeten Journalistin begeistert von ihren Erlebnissen erzählt hatte, erschien in einer Frauenzeitschrift ein Artikel mit dem Titel: „Brich Dein Bein im Bündnerland“. Von kollegialer Seite gingen nun giftige Pfeile gegen den unschuldigen Martin Allgöwer.
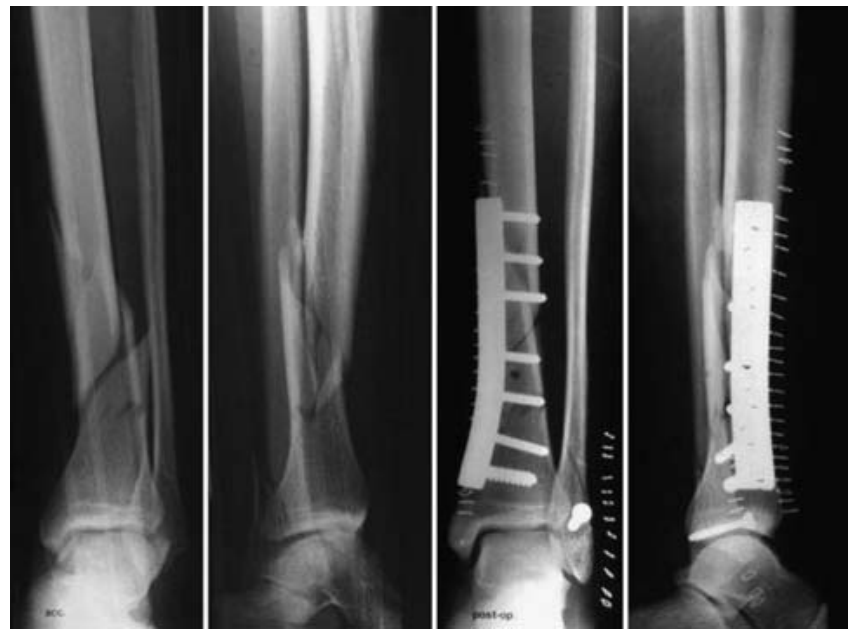

Abb. 39 Röntgenbilder nach Sportverletzung. Patient B.v.P.

Etwa 10 Jahre nachher entstehen neue Varianten:

Am Sonntagmorgen erklärte mir der am Vorabend operierte Gynäkologe aus Bayern: „Herr Kollege, ich trete am nächsten Mittwoch aus. Ich habe am Donnerstag ein eingeplantes großes Operationsprogramm. Da ich vaginal operiere und dabei sitze, entsteht am Bein keine Belastung“. Eine Art Erpressung?

\section{Hat die AO eine anerkannte oder gar leitende Position in der internationalen Traumatologie gewonnen?}

Viele jüngere, aktive Mitglieder neigen wahrscheinlich zu einer positiven Antwort. Das ist sicher eine Überschätzung.

Kommen wir auf unsere Säulen zurück: Die Veränderungen sind enorm.

- Was ist im Bereich Forschung (R) geschehen? Bei der Gründung war das unter chirurgischer Leitung stehende Davoser Laboratorium ein Unikat. Heute hat jede Universitätsklinik ihr eigenes Forschungsinstitut.

- Die Dokumentation (D). Das Verständnis und die Kraft für eine allgemeine Dokumentation der Resultate sind schnell verloren gegangen. Man beschränkt sich heute auf gezielte klinische Studien vor der Einführung neuer Techniken oder Implantate.

- Instrumentation (I) gewiss, die neue „Synthes“ ist jetzt eine weltumspannende mächtige Firma geworden und hat den Vorteil, dass sie vom Davoser Institut und einer sehr kompetenten technischen Kommission beraten wird. Andererseits offerieren schon lange Konkurrenzfirmen ähnliche, sehr gute Fabrikate nach wesentlich niedrigeren Entwicklungskosten.

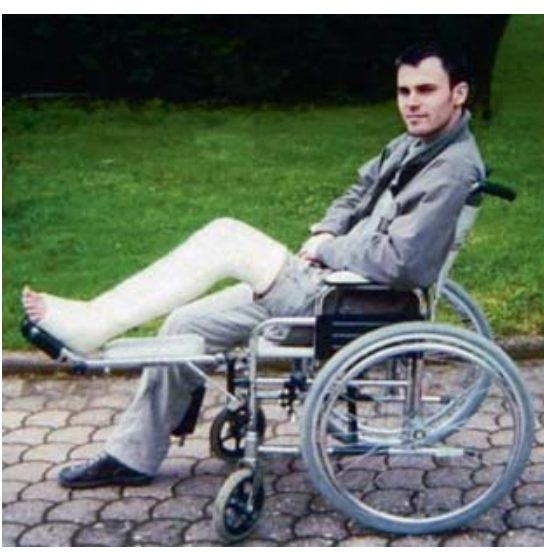

Abb. 40 Der Patient im Rollstuhl im langen Zirkulärgips mit gebeugtem Knie für 8 Wochen (Foto U. Heim, mit Genehmigung des Patienten).

- Noch ist der Unterricht $(\mathrm{T})$ in einer sehr guten Verfassung, vielleicht führend. Als ich einmal Willenegger sagte, mich störe es, festzustellen, wie oft Kunden anderer Produzenten sich in unseren Kursen ausbilden, meinte er: „Gute AO-Osteosynthesen sind nicht von Implantaten einer bestimmten Marke abhängig“. Entscheidend sei nur die Einhaltung der Grundregeln und das ist, nebst der Notfalloperation („8-Stundengrenze“), was er selbst schon an einem Vortrag an der SGUBTagung 1957 so definierte: „... nur im Rahmen einer auf das Funktionelle ausgerichteten Denkweise lässt sich die operative Frakturenbehandlung überhaupt ernsthaft begründen“ (Ph. 34).

Und genau in diesen 2 elementaren und komplementären Forderungen der $\mathrm{AO}$, nämlich primäre Stabilisierung des Skeletts und aktive schmerzfreie Bewegung aller Gelenke, hapert es. 


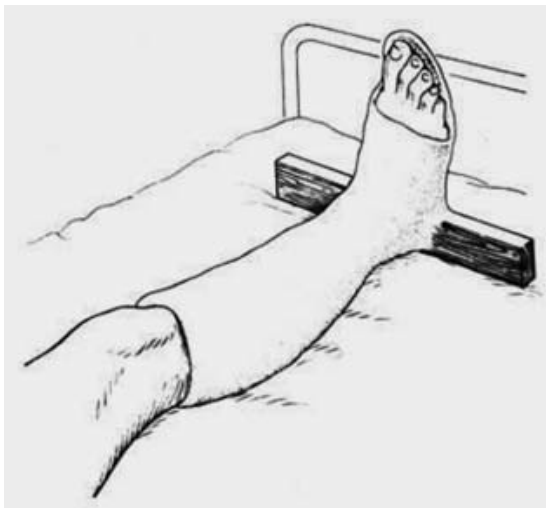

Abb. 41 Unverstandene AO-Prinzipien: Ruhigstellung nach instabilem Marknagel durch zusätzliche äußere Fixation im „Antirotationsstiefel“. Schweizerische Universitätsklinik (Aus: U. Heim, J. Baltensweiler, Checkliste Traumatologie. Stuttgart, New York: Thieme; 1981. S. 315, Abb. 306).

Natürlich verunmöglichen heute Komplexität und Schweregrad der Verletzung oft eine definitive Versorgung. Aber die 1 . notfallmäßige provisorische Stabilisierung bleibt. Die aktive Bewegung muss dann angepasst werden.

Doch auch beim „banalen“ Monotrauma klappt es oft nicht mehr. Strukturelle und personelle Änderungen im Krankenhauswesen sind mitverantwortlich.

Dafür nun ein charakteristisches Trendbeispiel, nicht etwa eine Ausnahme:

Dem 22-jährigen Sohn eines Freundes geschah in einer der größten Städte seines Landes Folgendes: er befand sich am Sonntag beim Rugbyturnier plötzlich ganz an den Boden gepresst, der große Haufen der Mitspieler über ihm, kämpfend. Er spürte das Krachen im Unterschenkel. Seine Freundin, Operationsschwester in einer Privatklinik, fand erst nach 4 Tagen einen Operateur - in einer anderen Klinik. Zum postoperativen Röntgenbild (Abb. 39) „no comment“.

Die Implantate sind wahrscheinlich Synthes. Einige Wochen später fand ich ihn
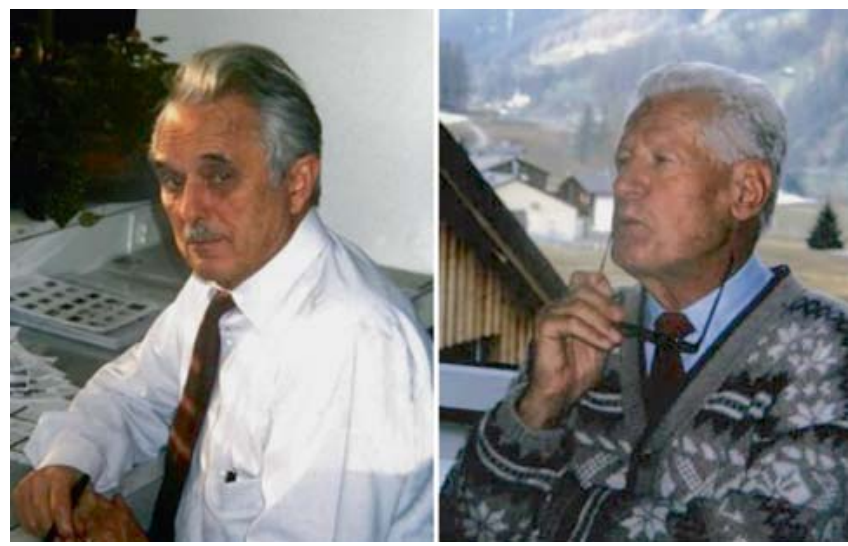

Abb. 42 Späte Fotografien von M.E. Müller und M. Allgöwer. Leider sind beide nicht mehr unter uns (Foto in Davos U. Heim, ca. 2005).

zuhause im Rollstuhl mit langem Zirkulärgips (Abb.40), verordnet für 8 Wochen.

Wegen des fixierten gebeugten Knies konnte er sich kaum bis in den Garten bewegen. Schlussendlich wurde dann doch alles gut, auch funktionell: er war jung. Aber eine Cerclage Modell 1950 hätte den gleichen Dienst getan, einfach und viel billiger.

Wahrscheinlich hat der Operateur der Stabilität auf Dauer nicht vertraut. Ich weiß, dass sehr viele den Gips „nicht weglassen können“. Es ist dies ein sehr altes, vererbtes Gefühl, eine Art psychische „Vergipsung“.

Oft werden die AO-Prinzipien an bedeutenden Kliniken nicht verstanden bzw. durchgedacht (Abb. 41).

Es ist das historische Verdienst der AO, bei stabilisiertem Skelett die aktive Mobilisation aller Gelenke durch Beispiel und Lehre verbreitet zu haben.

Diese elementare Leistung - so hoffen wir - werden auch die nachfolgenden Generationen vertreten. Die heute viel differenzierteren und gewebeschonenderen Synthesetechniken können der weiteren Optimierung dienstbar gemacht werden.

\section{Literatur}

${ }^{1}$ Heim UFA. Das Phänomen AO. Bern, Stuttgart, Toronto, Seattle: Hans Huber; 2001

2 Matti $H$. Die Knochenbrüche und ihre Behandlung. 2. Auflage. Berlin: Springer; 1931

3 Böhler L. Die Technik der Knochenbruchbehandlung. 12.-13. Auflage. Wien: Maudrich; 1953

${ }^{4}$ Böhler L. Technik der Knochenbruchbehandlung im Frieden und im Kriege, 3. Bd. Die Marknagelung nach Küntscher. Wien: Maudrich; 1945: 1822

${ }^{5}$ Lambotte A. L'intervention opératoire dans les fractures. Paris: Masson; 1907

${ }^{6}$ Lambotte A. Chirurgie opératoire des Fractures. Paris: Masson; 1913

7 Danis $R$. Théorie et pratique de l'ostéosynthèse. Paris: Masson; 1949

${ }^{8}$ König F. Operative Chirurgie der Knochenbrüche. Berlin: Springer; 1931

${ }^{9}$ Weber BG. Die Verletzungen des oberen Sprunggelenkes. Bern: Hans Huber; 1966

10 Müller ME, Allgöwer M, Willenegger $H$. Technik der operativen Frakturenbehandlung. Berlin, Göttingen, Heidelberg: Springer; 1963; Library of Congress Catalog Card Number 63-17394

${ }^{11}$ Müller ME, Allgöwer M, Willenegger $\mathrm{H}$. Manual der Osteosynthese. Berlin, Heidelberg, NewYork: Springer; 1969; Library of Congress Catalog Card Number 72-88818

12 Texhammar R, Colton Ch. Das AO Instrumentarium, 2. Auflage. Berlin, Heidelberg, New York: Springer; 1994

\section{Priv.-Doz. Dr. med. U. Heim}

Schänzlistraße 65

3013 Bern 